\title{
A Predictive Model of Enhanced Oil Recovery by Infill Drilling and Its Application
}

\author{
Xu Jianhong ${ }^{1}$, Cheng Linsong ${ }^{1}$ and Ma Lili ${ }^{2}$ \\ (1. School of Petroleum Engineering, China University of Petroleum, Beijing 102249, China) \\ (2. The Fourth Production Factory, Daqing Oilfield, Daqing, Helongjiang 163511, China)
}

\begin{abstract}
Infill drilling is now recognized as a viable improved recovery process. However, the reliable prediction of incremental recovery by infill drilling cannot be readily and accurately determined by present techniques. This paper proposes a hybrid predictive model of stream tube simulation and numerical simulation by using the contemporary theory of fluid flow in porous media. The model calculates the geometries of stream tubes, remaining oil distribution and water cut at different development stages in the near future, and uses a three-dimensional simulation to track fluid movement in each stream tube slice. This will help reservoir engineers to determine the feasibility of infill drilling. This predictive model is used to forecast the degree of control of well pattern, the ultimate incremental recovery of infill wells within an inverted 5-spot case in an oilfield and the economic benefit is also analyzed.
\end{abstract}

Key words: Predictive model, infill drilling, stream tube, numerical simulation, recovery process

\section{Introduction}

Infill drilling for increasing oil production and achieving incremental recovery has been a common industrial practice for many years. Infill drilling can improve reservoir continuity, areal sweep, vertical sweep, recovery of wedge edge oil, and reduce economic limits of a project (Barbe and Schnoebelen, 1987; Barber, et al., 1987). However, it is difficult to determine well spacing economically and effectively. Several researchers studied infill performance in oilfields (Fuller, et al., 1992; Wang, et al., 2004), and they defined optimum well spacing as the density of wells that will result in the greatest ultimate profit to the operator. Their research was concentrated in two aspects: 1) optimum number of wells; 2) and location of wells. The incremental recovery by infill drilling cannot be accurately determined by present models and techniques. Previous approaches involve overly simple stream tube models, or overly complex 3-D numerical simulators combined with separate economic analysis.

The infill drilling predictive model (IDPM) presented in this paper is a three-dimensional (stratified, five-spot), three-phase model, which uses a minimal amount of reservoir and geological data to generate oil production and recovery forecasts for ongoing water flood and infill drilling projects. According to the deliverability of infill well and predicted result of recovery efficiency, reservoir engineers can analyze and evaluate the feasibility of infill drilling program and its economic benefit.

\section{Infill drilling predictive model}

The IDPM is a combination of stream tube and numerical simulators, introduced separately in the following.

\subsection{Stream tube calculation}

\subsubsection{Pressure field}

When ignoring gravity and capillary potential, the pressure equation of a three-dimensional, three-phase system can be expressed as follows (Baek and Hewett, 2000; Wang, et al., 2004):

$$
\begin{gathered}
\nabla(\lambda \nabla p)=\phi C_{1} \frac{\partial p}{\partial t} \\
\lambda=\lambda_{\mathrm{o}}+\lambda_{\mathrm{w}}+\lambda_{\mathrm{g}} \\
=\left(\frac{K K_{\mathrm{ro}}}{\mu}\right)_{\mathrm{o}}+\left(\frac{K K_{\mathrm{rw}}}{\mu}\right)_{\mathrm{w}}+\left(\frac{K K_{\mathrm{rg}}}{\mu}\right)_{\mathrm{g}}
\end{gathered}
$$

where $\lambda$ is fluid mobility, $10^{-3} \mathrm{~mm}^{2} /(\mathrm{mPa} \cdot \mathrm{s}) ; C_{1}$ is compressibility, $10^{-6} \mathrm{MPa}^{-1} ; p$ is pressure; $\phi$ is porosity, dimensionless; $K$ is the absolute permeability of medium, $10^{-3} \mu \mathrm{m}^{2} ; K_{\mathrm{ro}}, K_{\mathrm{rw}}$ and $K_{\mathrm{rg}}$ are the relative permeability to oil, water, and gas, respectively, fraction; $\mu$ is fluid viscosity, $\mathrm{mPa} \cdot \mathrm{s}$; subscripts $\mathrm{o}, \mathrm{w}$, and g denote oil, water, and gas phase; subscript 1 represents o and w, i.e. oil and water phase.

Outer boundary conditions are:

$$
\nabla(\lambda h \nabla p)=F
$$


where $h$ is reservoir thickness; and $F$ denotes a source term.

For steady-state flow, $F=0$; for pseudosteady-state flow, $F=\phi \operatorname{ch}(\partial p / \partial n)$.

Eq. (3) is a Laplace equation if $F=0$, or a Poisson equation if $F \neq 0$, and can be solved by the finite element method. The solution of the Poisson equation is:

$$
\frac{\partial}{\partial x}\left(K \frac{\partial p}{\partial x}\right)+\frac{\partial}{\partial y}\left(K \frac{\partial p}{\partial y}\right)=F
$$

In each simulated grid, oil recovery $N_{\mathrm{p}}$, water cut $f_{\text {we }}$, and total flow rate, $q$ are expressed in dimensionless time. The total flow rate of the grid, $q$, is calculated by the following equation:

$$
q^{k}=\sum_{i=1}^{N_{1}} q_{i}^{k}=\sum_{i=1}^{N_{1}} \frac{\Delta p_{\mathrm{o}}}{R_{\mathrm{T} i}^{k}}
$$

where superscript $k$ is time step index and subscript $i$ is stream tube index; $N_{1}$ is the total number of stream tubes in the model.

From the definition of dimensionless variables, we have

$$
R_{\mathrm{Td} i}^{k}=\frac{R_{\mathrm{T} i}^{k}}{\Delta p_{\mathrm{o}} / q_{\mathrm{o} i}}
$$

Substituting Eq. (6) into Eq. (5) gives

$$
q^{k}=\sum_{i=1}^{N_{1}} \frac{q_{\mathrm{o} i}}{R_{\mathrm{Td} i}^{k}}
$$

where $\Delta p_{\mathrm{o}}$ and $q_{\mathrm{o} i}$ are constants; $R_{\mathrm{T} i}^{k}$ is total stream tube resistance in stream tube $i ; R_{\mathrm{Td} i}^{k}$ is total dimensionless stream tube resistance.

\subsubsection{Calculation of oil recovery and water cut}

Using a small time step, e.g., $\triangle t$, the cumulative injection, $Q_{m}$, is calculated in each grid as

$$
\begin{aligned}
& Q_{m}^{k}=Q_{m}^{k-1}+\Delta t^{k} q_{m}^{k-1} \\
& \left.\begin{array}{l}
Q_{m}^{k-1}=0 \\
Q_{m}^{k}=q_{\mathrm{o} m}
\end{array}\right\} \text { at } k=1
\end{aligned}
$$

where $q_{\mathrm{o} m}$ is the total flow rate of grid $m$ at the beginning; and $k$ is time step index..

For each producer, total fluid flow rate, $q_{\mathrm{T}}$, total water flow rate, $q_{\mathrm{w}}$, and water cut, $F_{\text {we }}$, can be expressed as follows:

$$
\begin{gathered}
q_{\mathrm{T}}^{k}=\sum_{m=1}^{N} q_{m}^{k} \\
q_{\mathrm{w}}^{k}=\sum_{m=1}^{N} q_{m}^{k} f_{\mathrm{we} m}^{k} \\
F_{\mathrm{we}}^{k}=\frac{q_{\mathrm{w}}^{k}}{q_{\mathrm{T}}^{k}}
\end{gathered}
$$

where $N$ is the total number of grids for the producer.

The field production and injection are calculated by simple summation.

$$
\begin{aligned}
& Q_{\mathrm{T}}^{k}=\sum_{m=1}^{M} Q_{m}^{k} \\
& N_{\mathrm{pT}}^{k}=\sum_{m=1}^{M} N_{\mathrm{p} m}^{k}
\end{aligned}
$$

where $Q_{\mathrm{T}}$ and $N_{\mathrm{pT}}$ are field cumulative injection and field cumulative production; $M$ is the total number of grids in the model. At the next time step $(k+1)$, the previous four steps are repeated.

\subsection{Reservoir simulation}

\subsubsection{Fundamental flow equations and their solutions}

The flow equations can be expressed as an equivalent but much simpler form (Fanchi, 2001).

For liquids (oil and water):

$$
-\nabla \cdot \frac{v_{1}}{B_{1}}-\frac{q_{1}}{\rho_{\mathrm{lsc}}}=\frac{\partial}{\partial t}\left(\phi S_{1} / B_{1}\right)
$$

For gas:

$$
\begin{aligned}
& -\nabla \cdot\left(\frac{v_{\mathrm{g}}}{B_{\mathrm{g}}}+\frac{R_{\mathrm{so}}}{B_{\mathrm{o}}} v_{\mathrm{o}}+\frac{R_{\mathrm{sw}}}{B_{\mathrm{w}}} v_{\mathrm{w}}\right)-\frac{q_{\mathrm{g}}}{\rho_{\mathrm{gsc}}} \\
& =\frac{\partial}{\partial t}\left[\phi\left(\frac{S_{\mathrm{g}}}{B_{\mathrm{g}}}+\frac{R_{\mathrm{so}} S_{\mathrm{o}}}{B_{\mathrm{o}}}+\frac{R_{\mathrm{sw}} S_{\mathrm{w}}}{B_{\mathrm{w}}}\right)\right]
\end{aligned}
$$

The Darcy velocities may be written as follows:

$$
\begin{aligned}
& v_{1}=-K \cdot \lambda_{1} \nabla \Phi_{1} \\
& =-\lambda_{1}\left[i K_{x} \frac{\partial \Phi_{1}}{\partial x}+j K_{y} \frac{\partial \Phi_{1}}{\partial y}+k K_{z} \frac{\partial \Phi_{1}}{\partial z}\right] \\
& v_{\mathrm{g}}=-K \cdot \lambda_{\mathrm{g}} \nabla \Phi_{\mathrm{g}} \\
& =-\lambda_{\mathrm{g}}\left[i K_{x} \frac{\partial \Phi_{\mathrm{g}}}{\partial x}+j K_{y} \frac{\partial \Phi_{\mathrm{g}}}{\partial y}+k K_{z} \frac{\partial \Phi_{\mathrm{g}}}{\partial z}\right]
\end{aligned}
$$


where $v_{1}$ is the flow velocity of phase $1, \mathrm{~m} / \mathrm{s} ; v_{\mathrm{g}}$ is the flow velocity of gas phase, $\mathrm{m} / \mathrm{s} ; B$ is formation volume factor, fraction; $\rho_{\text {ssc }}$ and $\rho_{\text {gsc }}$ are the densities of phase 1 and gas phase in standard conditions, $\mathrm{kg} / \mathrm{m}^{3} ; S$ is saturation; $R_{\mathrm{so}}$ and $R_{\mathrm{sw}}$ are dissolution gas-oil ratio and dissolution gas-water ratio, $\mathrm{m}^{3} / \mathrm{m}^{3} ; \Phi$ is fluid potential, $\Phi_{\mathrm{o}}=p_{\mathrm{o}}-g \rho_{\mathrm{o}} D, \quad \boldsymbol{\Phi}_{\mathrm{w}}=p_{\mathrm{w}}-g \rho_{\mathrm{w}} D, \quad \boldsymbol{\Phi}_{\mathrm{g}}=p_{\mathrm{g}}-g \rho_{\mathrm{g}} D ;$ and $D$ is reservoir depth, $\mathrm{m}$.

\subsubsection{Pressure equation}

Pressure equation can be written as:

$$
\begin{aligned}
& p_{1}=\frac{\phi}{B_{1}} \frac{\partial S_{1}}{\partial t}+\left[\frac{S_{1}}{B_{1}} \frac{\partial \phi}{\partial p_{1}}-\frac{S_{1} \phi}{B_{1}{ }^{2}} \frac{\partial B_{1}}{\partial p_{1}}\right] \frac{\partial p_{1}}{\partial t} \\
& p_{\mathrm{g}}=\left(\frac{\phi R_{\mathrm{so}}}{B_{\mathrm{o}}}-\frac{\phi}{B_{\mathrm{g}}}\right) \frac{\partial S_{\mathrm{o}}}{\partial t}+\left(\frac{\phi R_{\mathrm{sw}}}{B_{\mathrm{w}}}-\frac{\phi}{B_{\mathrm{g}}}\right) \frac{\partial S_{\mathrm{w}}}{\partial t} \\
& +\left\{\frac{S_{\mathrm{g}}}{B_{\mathrm{g}}} \frac{\partial \phi}{\partial p_{\mathrm{o}}}-\frac{S_{\mathrm{g}} \phi}{B_{\mathrm{g}}{ }^{2}}+\frac{B_{\mathrm{g}}}{\partial p_{\mathrm{o}}}+\frac{S_{\mathrm{o}} R_{\mathrm{so}}}{B_{\mathrm{o}}} \frac{\partial \phi}{\partial p_{\mathrm{o}}}\right. \\
& +\frac{\phi S_{\mathrm{o}}}{B_{\mathrm{o}}} \frac{\partial R_{\mathrm{so}}}{\partial p_{\mathrm{o}}}-\frac{\phi S_{\mathrm{o}} R_{\mathrm{so}}}{B_{\mathrm{o}}{ }^{2}} \frac{\partial B_{\mathrm{o}}}{\partial p_{\mathrm{o}}}+\frac{S_{\mathrm{w}} R_{\mathrm{sw}}}{B_{\mathrm{w}}} \frac{\partial \phi}{\partial p_{\mathrm{o}}} \\
& \left.+\frac{\phi S_{\mathrm{w}}}{B_{\mathrm{w}}} \frac{\partial R_{\mathrm{sw}}}{\partial p_{\mathrm{o}}}-\frac{\phi S_{\mathrm{w}} R_{\mathrm{sw}}}{B_{\mathrm{w}}{ }^{2}} \frac{\partial B_{\mathrm{w}}}{\partial p_{\mathrm{o}}}\right\} \frac{\partial p_{\mathrm{o}}}{\partial t}
\end{aligned}
$$

\subsubsection{Definite conditions}

1) Boundary condition:

$$
\left.\frac{\partial p}{\partial \sum}\right|_{\Sigma \in f(x, y, z)}=0
$$

2) Initial condition:

$$
p_{i}(x, y, z, 0)=p_{i}(x, y, z)
$$

\subsubsection{Subsidiary conditions}

1) Saturation equation:

$$
S_{\mathrm{o}}+S_{\mathrm{w}}+S_{\mathrm{g}}=1
$$

2) Capillary pressure:

$$
\begin{gathered}
p_{\mathrm{g}}=p_{\mathrm{o}}+p_{\mathrm{cgo}} \\
p_{\mathrm{w}}=p_{\mathrm{o}}-p_{\text {cow }}
\end{gathered}
$$

3) Equation of state:

Relative permeability

$$
\begin{gathered}
K_{\mathrm{ro}}=K_{\mathrm{ro}}\left(S_{\mathrm{g}}, S_{\mathrm{w}}\right) \\
K_{\mathrm{rg}}=K_{\mathrm{rg}}\left(S_{\mathrm{g}}\right) \\
K_{\mathrm{rw}}=K_{\mathrm{rw}}\left(S_{\mathrm{w}}\right)
\end{gathered}
$$

Capillary pressure:

$$
\begin{gathered}
p_{\text {cgo }}=p_{\text {cgo }}\left(S_{\mathrm{g}}\right) \\
p_{\text {cow }}=p_{\text {cow }}\left(S_{\mathrm{w}}\right)
\end{gathered}
$$

Fundamental flow equations, pressure equation and definite conditions form the entire mathematical equation, which can be solved by using the finite difference method combining subsidiary conditions.

\section{Model performance}

To illustrate the model proposed, a simple application where water was injected into a low permeability oil reservoir was presented. In the present work, it was considered that wells were arranged in a quarter 5-spot pattern with different permeability distribution. After infilling drilling, the 5-spot pattern was changed to 9-spot pattern, which had one injector and eight producers, as shown in Fig. 2. The calculated results indicated that the stream tubes were only concentrated near the main stream lines between producers and injector before infill drilling (Fig. 1), but after infill drilling the stream tubes controlled the whole well pattern, and the water flood swept region increased greatly (Fig. 2). The recovery efficiency increased by $4.54 \%$ after infill drilling.

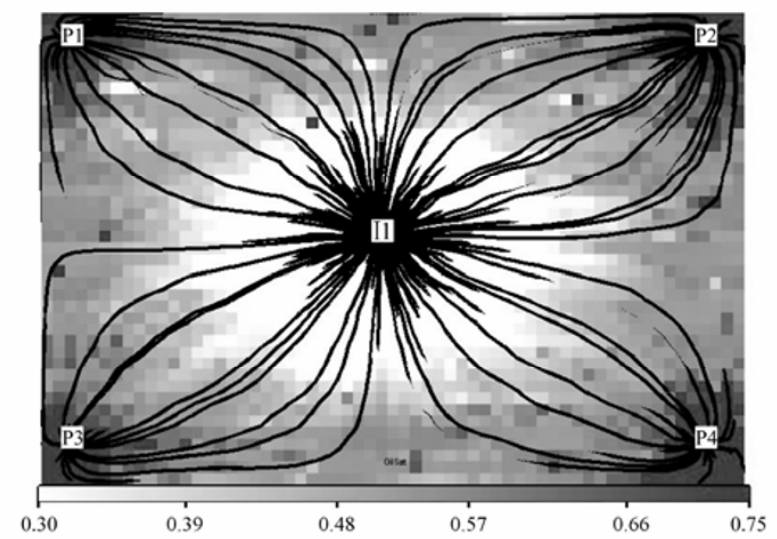

Fig. 1 The stream tube geometry and remaining oil saturation distribution for a quarter inverted 5-spot case (Before infill drilling) 


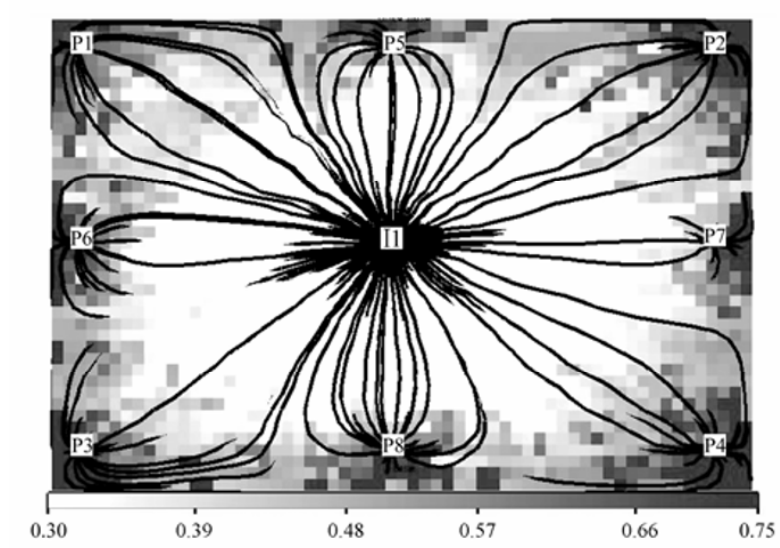

Fig. 2 The stream tube geometry and remaining oil saturation distribution for a inverted 9-spot case

(After infill drilling)

\section{Economic benefit analysis}

The economic benefit from infill drilling can be deduced by using the input/output rule:

$$
\begin{aligned}
& M=\Delta R \cdot N_{\mathrm{o}} \cdot R_{\mathrm{c}}\left(P_{\mathrm{o}}-C_{\mathrm{p}}\right) \\
& -n \cdot\left(D_{\mathrm{v}} \cdot C_{\mathrm{v}}+C_{\mathrm{fh}}\right)(1+R)^{T / 2}-C_{\text {other }}
\end{aligned}
$$

where $N_{\mathrm{o}}$ is oil in place, $10^{4} \mathrm{t} ; R_{\mathrm{c}}$ is the commodity ratio of crude oil, fraction; $P_{\mathrm{o}}$ is the after-tax oil price, $10^{4} \mathrm{Yuan}(\mathrm{RMB}) / \mathrm{t} ; n$ is the number of infill wells; $D_{\mathrm{v}}$ is well depth, $\mathrm{m} ; C_{\mathrm{v}}$ is the drilling cost per meter, $10^{4}$ Yuan $/ \mathrm{m} ; C_{\mathrm{fh}}$ is the ground construction cost per well, $10^{4}$ Yuan/well; $C_{\mathrm{p}}$ is oil production cost, $10^{4}$ Yuan/t; $C_{\text {other }}$ is other expenses, $10^{4}$ Yuan; and $R$ is the investment lending rate, fraction.

Example The following reservoir properties and economic exponents are as follows: $\Delta R=4.536 \%, N_{\mathrm{o}}$ $=91.2 \times 10^{4} \mathrm{t}, R_{\mathrm{c}}=0.95, n=4, D_{\mathrm{v}}=3000 \mathrm{~m}, C_{\mathrm{v}}=1050$ Yuan $/ \mathrm{m}, C_{\mathrm{fh}}=120 \times 10^{4}$ Yuan, $C_{\mathrm{p}}=300$ Yuan $/ \mathrm{t}, R=$ 0.05 . Substituting the above parameters into Eq. (31) gives the economic benefit of four infill wells i.e. 77.76 million Yuan, so the infill development program is economically and technically feasible.

\section{Conclusions}

1) A hybrid predictive model is proposed by combining stream tube simulation with numerical simulation. The liquid, oil and gas withdrawal rates can be calculated, and the movement of fluid can be traced by using the hybrid predictive model.

2) The variation of oil saturation distribution after infill drilling can be predicted, the stream tube distribution and the degree of control of water flood can be analyzed by using the hybrid predictive model. The technical feasibility of infill drilling program can be determined by evaluating infill drilling effectiveness.

3) According to the infill drilling effectiveness, the economic benefit can be evaluated, and the economic feasibility of infill drilling program can be determined.

\section{References}

Baek M. and Hewett T. A. (2000) A hybrid stream tube simulator using a semi-analytical method. SPE Annual Technical Conference and Exhibition held in Dallas, Texas, 1-4 October 2000. SPE paper 63151

Barbe J. A. and Schnoebelen D. J. (1987) Quantitative analysis of infill performance: Robertson Clearfork Unit. Journal of Petroleum Technology, (December), 1593-1601 (SPE paper 15568)

Barber Jr. A. H., George C. J., Stiles L. H. and Thompson B. B. (1983) Infill drilling to increase reserves: Actual experience in nine fields in Texas, Oklahoma, and Illinois. Journal of Petroleum Technology, (August), 1530-1538 (SPE paper 11023)

Fanchi J. R. (2001). Principles of Applied Reservoir Simulation. New York: Gulf Professional Publishing, 31-55

Fuller S. M., Sarem A. M. and Gould T. L. (1992) Screening waterfloods for infill drilling opportunities. International Meeting on Petroleum Engineering held in Beijing, China, 24-27 March 1992. SPE paper, 22333

Wang H. B., Su Z. G. and Chen Z. Y. (2004). 3-D streamline model in water flooding reservoir. Beijing: Petroleum Exploration and Development, 32(2), 99-103 (in Chinese)

\section{About the first author}

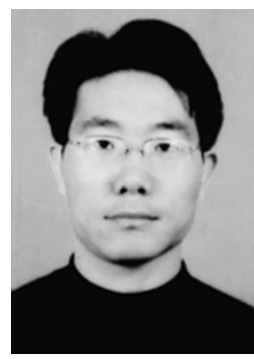

Xu Jianhong was born in 1978 and received his MS degree from China University of Petroleum (Beijing) in 2005. Now he is a doctor candidate in the same school with his research interests in reservoir engineering and reservoir simulation.

E-mail: xujhmail@163.com

(Received September 22, 2006) (Edited by Sun Yanhua) 